einigen Unzufriedener in der SPLM/A-Führung, die sich von Garangs autoritärem Führungsstil an den Rand gedrängt fühlen.

\title{
Vorboten eines Bürgerkrieges
}

Garangs Position wird aber weniger vom Regierungswechsel im Sudan denn vom Regierungswechsel in Äthiopien erschüttert. In den späten 1980er-Jahren wird die Position des Derg-Regimes gegenüber verschiedenen Rebellenorganisationen, insbesondere aus dem nördlichen Tigray, prekär. Die SPLA kann sich einer unmittelbaren Verwicklung in diese Kämpfe nicht entziehen. SPLA-Kontingente unterstützen, nicht zuletzt aus einer Art historischer Bringschuld, den Abwehrkampf der Derg militärisch. Diese eindeutige politisch-militärische Positionierung führt nach dem endgültigen Sturz des Derg-Regimes im Mai $1991 \mathrm{zu}$ unmittelbar spürbaren negativen Einschnitten. Der Weg zu Verhandlungen mit den neuen Machthabern, der Rebellenallianz Ethiopian People's Revolutionary Democratic Front (EPDRF) unter dem neuen Präsidenten Meles Zenawi ist verbaut. Die militärische Allianz bricht zusammen. "Zenawi immediately cut off all arms shipments to Garang, shut down the SPLA bases in western Ethiopia, and ended the training and support programs. [...] The external base of support for Garang's war disappeared in a single moment.« (Natsios, 2012: 97)

Der Umsturz in Äthiopien führt auch zu humanitären Verwerfungen. In Gambela, der äthiopischen Region an der Grenze zu Südsudan, kommt es zu massiven Fluchtbewegungen, was zu einer Verschlechterung der Nahrungsmittelsituation in Jonglei auf der südsudanesischen Seite führt. Khartum verbietet OLS und dem in ihrem Rahmen operierenden World Food Programme (WFP), humanitäre Güter in dieser Kernregion des bewaffneten Widerstandes zu liefern. Dies setzt wiederum die SPLM/A unter beträchtlichen Handlungsdruck, insbesondere im konkreten Umgang mit den Geflüchteten.

Innerorganisatorisch wirkt sich der äthiopische Machtwechsel fatal aus. Garangs Führungsposition ist weniger aufgrund seine persönlichen Leadership-Qualitäten unumstritten. Sein Hang zu harschen und mitunter despotischen Entscheidungen wird von den meisten führenden Exponent:innen der Bewegung seit der Gründung der SPLM/A kritisiert (Rolandsen und Daly, 2016: 121). Es ist vielmehr seine persönliche Verbundenheit mit der für die Organisation lebenswichtigen äthiopischen Unterstützung, die Garangs Unantastbarkeit erklärt. Und diese Unterstützung geht nun verloren. »As 
long as Garang had Mengistu's support he was able to exert considerable control over the Movement's hierarchy.« (Johnson, 2016a: 91)

Am 28. August 1991 lancieren die drei führenden SPLM-Kader Riek Machar Teny, Lam Akol Ajawin und Gordon Kong Chuol in Nasir in Upper Nile einen Putsch gegen Garang. Die erste Nachricht der Putschisten über das SPLM/A-Radionetzwerk lässt an Deutlichkeit nichts zu wünschen übrig:

»For the last eight years, John Garang has been running the movement in a most dictatorial and autocratic manner. He oppressed, humiliated and degraded the people and turned a popular struggle into war-lordism and a reign of terror. [...] In order to save the movement from the imminent collapse, it has been decided to relieve John Garang from the leadership of SPLM/A.« (zitiert nach Akol, 2003: 291)

Ort und Datum sind nicht zufällig gewählt. Den Rebellen ist wohl bewusst, dass sich zum Zeitpunkt ihres internen Putsches eine große Zahl an internationalem Hilfspersonal und UN-Mitarbeiter:innen in Nasir befinden (vgl. die Aussagen des am Putsch selbst federführend beteiligten Lam Akol, 2003: 12). Diese Kontakte können umgehend für den Aufbau einer eigenen Hilfsstruktur verwendet werden, ein entscheidendes Kriterium für die Gewinnung internationaler Anerkennung und Unterstützung.

Wiewohl OLS und die vorangegangenen Hilfskampagnen in einem derart komplexen Umfeld immer beträchtliche Schwierigkeiten haben, Neutralität zu bewahren und nicht zu einem Teil des Konfliktes zu werden, nimmt die Politisierung von Hilfe mit dem Nasir-Split eine neue Dimension an. Garang verdächtigt die OLS sofort, mit den Partei-Rebellen zu kooperieren. Tatsächlich spielt die Zusammenarbeit mit Hilfsorganisationen eine fundamentale Rolle, einerseits in der Sicherung von lebenswichtigen Gütern, andererseits im Kampf um internationale Akzeptanz und Anerkennung. Nicht zufällig verlangt der erste Radiospot der Nasir-Rebellen eine Verstärkung der Zusammenarbeit mit der internationalen Hilfsoperation: The SPLM shall give more impetus to the relief efforts provided by the operation life-line Sudan, I.C.R.C. and other international relief agencies in Southern Sudan.« (Zitiert nach Akol, 2003: 292)

Machar gründet unmittelbar nach dem Nasir-Split und der Formierung der SPLM/A-Nasir - die Garang-Fraktion erhält in diesen Jahren schnell den Beinamen SPLM/A-Torit - die Rehabilitation Association of South Sudan (RASS). Die RASS positioniert sich im Ringen um die Kontrolle internationaler Hilfe in Konkurrenz zur schon 1984 gegründeten humanitären Organisation der 
SPLM/A, der Sudan Reliefand Rehabilitation Association (SRRA). Die Leitung der RASS wird vom Machar-Vertrauten Taban Deng Gai übernommen, der die Funktion bald zum Aufbau einer strategischen Kooperation mit der NCPRegierung in Khartum nützt (Johnson, 2016a: 96, 99). Machar etabliert zudem gute persönliche Kontakte zu der in Nasir konzentrierten Hilfsindustrie. So heiratet er wenig später die britische humanitäre Helferin Emma McCune, die in den kurzen zwei Jahren der Ehe - McCune stirbt im Jahr 1993 in Nairobi bei einem Verkehrsunfall - zu einem wichtigen und international gehörten Sprachrohr von Machar wird.

Neben dem persönlichen Angriff auf Garang fokussiert der Nasir-Split auf den alten politischen Zielkonflikt des südlichen Widerstandes: gesamtsudanesische Revolution oder südsudanesische Unabhängigkeit. Dieser Zielkonflikt entwickelt sich allerdings erst allmählich. In ihren ersten Stellungnahmen bekennen sich die Rebellen zu einem »united secular Sudan« (Akol, 2003: 312). Diese Position reift im Verlauf des Jahres 1992 zur Propagierung einer Referendum-Lösung, die dann tatsächlich zu einem Ankerpunkt aller folgenden Verhandlungsansätze wird. Diese vorerst unklare politische Orientierung schlägt sich in taktischen Differenzen innerhalb der Nasir-Gruppe nieder. Während Riek Machar und Gordon Kong eine dauerhafte Abspaltung einer neuen organisatorischen Kraft favorisieren, will Lam Akol die Einheit der SPLM/A beibehalten und deren Führung übernehmen.

Die Kämpfe zwischen den Fraktionen beginnen praktisch unmittelbar nach der Abspaltung. Machars Truppen absorbieren einige der noch eigenständig operierenden Anya-nya II Elemente, die sich mit der von Nuer dominierten ethnopolitischen Konfiguration der SPLM/A-Nasir besser anfreunden können. Der erste Schwerpunkt der Kämpfe liegt in Jonglei, der geografischen Mitte zwischen Nasir im Nordosten und Torit im Südosten des südlichen Sudan. Zum Kulminationspunkt dieser mit äußerster Härte geführten bewaffneten Auseinandersetzungen wird das Bor Massaker am 15. November 1992. Von Machar geführte, Nuer-dominierte Verbände der SPLANasir und die mit ihnen verbündete Miliz der Nuer White Army attackieren um Bor lebende Dinka-Gemeinschaften mit kompromissloser Brutalität. 2.000 Menschen sterben, Zehntausende werden vertrieben. Das Massaker wird zu einem Fanal dieser Vorphase des südsudanesischen Bürgerkrieges. Es bleibt in den folgenden Jahrzehnten latent im politischen Diskurs präsent. Im April 2012 sieht sich der zu diesem Zeitpunkt bereits als Vizepräsident des unabhängigen Südsudan fungierende Riek Machar genötigt, sich öffentlich in Bor für die Ereignisse zu entschuldigen. 
Die Kämpfe zwischen den Fraktionen breiten sich in der Folge auf die Regionen westlich des Nil aus. Die SPLM/A-Nasir genießt in den von Nuer und Shilluk - der Gemeinschaft von Lam Akol - bewohnten Regionen von Unity und Upper Nile, traditionellen Problemgebieten der SPLM/A, die stärkste Unterstützung (Rolandsen, 2005: 38).

Der Nasir-Split markiert einen Wendepunkt in der Geschichte der SPLM/A. Diese war ursprünglich angetreten, die durch divergierende Gemeinschaftsinteressen und Stammes-Milizen segmentierte Widerstandsbewegung unter einem einheitlichen politisch-militärischen Programm zusammenzuführen. Mit dem Nasir-Split schlägt dieser Versuch nun in eine offene Ethnopolitisierung um (vgl. auch Jok, 2017; Hutchinson, 2000). Es geht nicht mehr um spezifische Interessenlagen einzelner Gemeinschaften, Gefühle von strategischer Benachteiligung oder simple Probleme der technischen Kooperation, wie lange Distanzen ohne entsprechende Transportinfrastruktur oder sprachliche Barrieren. Diese neue Form der Ethnopolitisierung resultiert in einer direkten und gewaltsamen Auseinandersetzung um Macht entlang identitätspolitischer Linien.

In der Auseinandersetzung mit der Regierung in Khartum wirft die Periode nach der Spaltung von 1991 bis 1994 den südsudanesischen Widerstand um Jahre zurück. Die sudanesische Armee fokussiert ihre Angriffe auf Regionen, die die SPLM/A aufgrund ihrer internen Konflikte vernachlässigt. Zu einer immer wichtiger werdenden Strategie, die die ohnehin schon prävalente Ethnopolitisierung verschärft, entwickelt sich die Kooperation mit lokalen Milizen, insbesondere in Equatoria. Auch die in Norduganda operierende und zu diesem Zeitpunkt militärisch starke Lord's Resistance Army (LRA) von Joseph Kony wird in diese Strategie miteinbezogen (LeRiche und Arnold, 2013: 91). Die sudanesische Unterstützung für die LRA währt über mehrere Jahre und führt sogar dazu, dass die LRA im Jahr 1994 eine Basis in Eastern Equatoria etabliert (Schomerus, 2007). Zugleich versucht Khartum politisch, die NasirFraktion für separate Friedensverhandlungen zu gewinnen.

Die militärische Antwort der SPLM/A-Torit ist die »Operation Jungle Storm«. Sie zielt 1992 spektakulär auf die Einnahme von Juba ab, scheitert aber ebenso spektakulär. Es bleibt nur der Rückzug in den Dschungel um die Imatong Mountains in Eastern Equatoria, der für einige Zeit der Ausgangspunkt der militärischen SPLA-Offensiven wird. Den ersten militärischen Erfolg nach dem Nasir-Split erringt die SPLA in der Region um den Aswa River nahe Nimule an der ugandischen Grenze gegen mit der sudanesischen Armee verbündete Milizen. In weiterer Folge kann die Grenzregion zu 
Uganda wieder eingenommen und nachhaltig kontrolliert werden. Diese aus der Not geborene Fokussierung auf den Süden bricht mit der langjährigen Vernachlässigung von Equatoria, was der SPLM/A politisch bald nutzen wird.

In dieser militärisch schwierigen Phase versucht die SPLM/A-Torit auch auf politischem Weg, die Initiative zurückzugewinnen. Sie beteiligt sich an einer letztendlich ergebnislosen Mediationsoffensive der OAU in Abuja (Young, 2012: 81) und kündigt für das Jahr 1994 ihre erste nationale Versammlung an, die »National Convention of New Sudan«. Ein von Lam Akol für die SPLM/A rekrutierter Garang- und späterer Kiir-Loyalist aus Equatoria (Abol Kuyok, 2015: 642-643), James Wani Igga, übernimmt die Vorbereitung der Versammlung, was die neue Verbindung der Organisation mit den südlichen Regionen unterstreicht. Nach einer Verschiebung findet die National Convention im April 1994 in Chukudum in Eastern Equatioria statt. 516 Delegierte aus allen Teilen des Sudan nehmen daran teil (Rolandsen, 2005: 101).

Tatsächlich gelingt es Garang mit dieser Veranstaltung, die politische Initiative zurückzugewinnen und der durch den gewaltsamen Spaltungsprozess tief verunsicherten Organisation neuen Optimismus zu vermitteln. »In general, the NC has become the symbol of a new start, when old errors were admitted and new practices adopted.«(Rolandsen, 2005: 111) Eine dieser neuen Praktiken ist die Rehabilitierung lokal verankerter Governance-Praktiken, insbesondere die Rolle der Chiefs, was die zivile SPLM-Administration flexibler und bei vielen Gemeinschaften populärer macht.

Die konkreten Resultate der National Convention sind in Hinblick auf die politisch-militärische Zielrichtung der Organisation ambivalent. Sie schlagen eine pragmatische Brücke zwischen Separatismus und der von Garang favorisierten »New Sudan«-Orientierung. So bekennt die erste Resolution des finalen Dokuments: »We, the people of the New Sudan, represented by this Convention, proclaim the birth of the New Sudan, which for the time being, shall consist of Bahr el Ghazal, Equatoria, Southern Blue Nile, Southern Kordofan and Upper Nile Regions«.

Diese territoriale Festlegung ist in doppelter Hinsicht bemerkenswert. Sie benennt den »New Sudan«, während sie gleichzeitig den proklamierten Südsudan in seiner maximalen Ausdehnung umreißt. Die diese Ambivalenz überbrückende Formulierung, »for the time being «, ist ausgesprochen vage gehalten. Die gesamtsudanesische Orientierung in der politischen Strategiebestimmung kommt in einem klaren Bekenntnis zum Engagement in der NDA zum Ausdruck, allerdings in einer ebenso ambivalenten Formulierung: "The National Convention expects the NDA to support the right to self-determi- 
nation for the oppressed people of the New Sudan and other marginalized areas«. Obwohl die NDA als das zentrale Bindeglied zwischen der SPLM/A und der nordsudanesischen Opposition fungiert, wird hier eine klare Erwartungshaltung formuliert: Im Austausch für ein Engagement in der NDA wird deren Unterstützung für eine mögliche Unabhängigkeit erwartet.

In der Nachbetrachtung kann diese Ambivalenz als eine bewusste Strategieentscheidung Garangs betrachtet werden. »Garang liked to show his Venn diagrams of different configurations of power and territory. These were options that he kept open, though he made it clear that he was committed to unity, with the SPLM wielding power in a manner that transformed Sudan as a whole. (de Waal, 2021: 328) Ob es sich dabei um ein gezieltes machtpolitisches Manövrieren in einem hochvolatilen politischen Umfeld gehandelt hat oder die Ursache in der Persönlichkeit Garangs, »a complex individual, with very complex motivations« (Malwal, 2015: 157), selbst gelegen war, wird wohl nie zu klären sein.

Während sich die SPLM/A-Torit mit dieser National Convention neu formiert, gerät die SPLM/A-Nasir in strukturelle Probleme. Mit Riek Machar und Lam Akol überwerfen sich deren beide Hauptexponenten, nachdem sich ihre unterschiedlichen taktischen Präferenzen als unvereinbar herausstellen. Lam Akol gründet die SPLM/A-United, um seinen Führungsanspruch für die gesamte Bewegung zu untermauern. Zugleich kämpft er jedoch wegen seiner publik werdenden Kontakte mit der Regierung in Khartum mit Absetzbewegungen. Riek Machar wiederum formiert um die von ihm präferierte Unabhängigkeitsstrategie eine neue Organisation, das South Sudanese Independence Movement (SSIM). Er trifft dabei auf substanzielle organisatorische Schwierigkeiten. Die Zersplitterung seiner anvisierten ethnopolitischen Basis, der $\mathrm{Nu}$ er, kann verhindert werden. Vielmehr kommt es innerhalb von Nuer-Milizen zu Spannungen, die Douglas Johnson (2016) als »Nuer Civil War« bezeichnet.

Der Nuer-Bürgerkrieg entflammt in den nördlichen Teilen von Greater Upper Nile. Die politischen Spannungen eskalieren gegen Ende der 1990erJahre. Auslöser ist im September 1997 die Nichtwahl eines der Hauptexponenten der SPLM/A-Nasir in Unity State, Paulino Matip Nhial, zum SPLM/ANasir-Gouverneur für Unity State. Matip, der die Bul Nuer-Einheiten um Bentiu kontrolliert, stellt sich mit seiner Miliz umgehend gegen Machar und den in der Region gut vernetzten Riek Gai Kok, der formal mit der Koordination des humanitären Sektors der SPLM/A-Nasir beauftragt ist (Abol Kuyok, 2015: 1007). Auch wenn diese spezifische Eskalation bald beigelegt werden kann, offenbart sie Machars politisch-militärische Schwäche. Er scheitert an 
der Herstellung einer ethnopolitischen Nuer-Allianz, die er strategisch in den Konflikt mit der SPLM/A-Torit hätte einbringen können. Die Auseinandersetzungen zwischen den Nuer-Milizen entfalten vielmehr eine Brutalität, die selbst abgehärtete SPLM/A-Nasir-Kommandanten wie Peter Gadet Yak dazu motiviert, sich wieder der SPLM/A-Hauptfraktion anzuschließen.

Die wechselvollste Geschichte der historischen SPLM/A-Führung durchlebt Kerubino Bol. Zunächst schließt er sich Akols SPLM/A-United an, um von dieser wegen zu enger Kontakte zum Regime in Khartum wieder entlassen zu werden. Aufbauend auf seiner starken militärischen Verankerung im nördlichen Bahr el-Ghazal gründet er die kurzlebige SPLM/A Bahr el Ghazal Faction. Diese Fraktion dient allerdings primär als Instrument seiner Wiederannäherung an die hauptsächlichen Spieler des Widerstandes. In den verbleibenden zwei Jahren seines Lebens wechselt er mehrfach die Seiten. Am spektakulärsten ist die von ihm kommandierte Einnahme von Wau, die von den SAF und Machar unterstützt wird. Er nutzt diesen Erfolg, um aus dieser Position der Stärke seine Reintegration in die SPLM/A zu verhandeln (Madut-Arop, 2006: 324-325). Doch er kann mit Garang keine Einigung erzielen. Letztlich wird er in Gefechten zwischen konkurrierenden Nuer-Milizen in Bentiu im September 1999 getötet. Vermutlich war er gerade im Begriff, eine neue Miliz für den Kampf gegen die SPLM/A in Bahr el-Ghazal zu formieren (Abol Kuyok, 2015: 668).

Aus einer Position der strategischen Schwächung, die durch den Aufschwung der SPLM/A-Torit im Anschluss an die National Convention verstärkt wird, entschließt sich Machar zu einer riskanten politische Offensive und tritt in deklarierte Friedensverhandlungen mit dem sudanesischen Regime ein. Im April 1996 unterschreibt er in Khartum, zusammen mit Kerubino Bols Fraktion, die sogenannte Political Charter. ${ }^{2}$ Diese Charter beinhaltet einen für Machars politische Position weitreichenden Kompromiss: die Akzeptanz staatlicher Einheit und der Scharia für das gesamte Staatsgebiet im Austausch für die Perspektive eines möglichen Unabhängigkeitsreferendums.

»2. The unity of the Sudan with its known boundaries shall be preserved [...]

3. [...] at the end of the interim period, a referendum shall be conducted by the people of the southern Sudan to determine their political aspirations. [...] 6. Sharia and custom shall be the sources of legislation. However, states 
may enact legislation complementary to the federal law in matters peculiar to those states. «

Dieser politische Abtausch wird in größerem Rahmen wenig später, im April 1997, im Khartoum Peace Agreement ${ }^{3}$ festgeschrieben. Dieses Abkommen mit dem sudanesischen Regime unterzeichnen neben Machars SSIM/A wiederum Kerubino Bols SPLM/A Bahr el Ghazal Faction, die in den Worten von Douglas Johnson als sudanesischer Proxy fungiert (Johnson, 2016a: 122), und zwei kleinere Rebellenbewegungen, die Equatoria Defence Force und die South Sudan Independents Group. Auch wenn diese Serie an von mit Khartum liierten SPLM/A-Minderheitsfraktionen betriebenen Abkommen in ihrer unmittelbaren Auswirkung auf das Geschehen minimal ist, entfalten sie dennoch nachhaltige Traktion. Sie handeln eine sequenzielle Formel der politischen Transition aus, die in alle folgenden Abkommen, bis hin zum im Jahr 2018 unterzeichneten R-ARCSS, eingeschrieben wird: Vereinbarung zu einem Waffenstillstand, Einleitung einer Interimsphase mit substanziellen Elementen einer Sicherheitssektorreform, und schlussendlich ein Status-Referendum (das in den Abkommen nach der Unabhängigkeit durch Wahlen ersetzt wird).

Das Khartoum Peace Agreement sieht für diese Interimsperiode vier Jahre vor. Währenddessen soll die militärische Sicherheit im Süden von einer eigenen Streitmacht wahrgenommen werden, der South Sudan Defence Force (SSDF), in die Machar seine SSIM/A-Truppen einzubringen beabsichtigt. Faktisch sollte die SSDF als ein deklariert südsudanesisches Instrument Khartums funktionieren, ist doch die primäre »Sicherheitsbedrohung«, gegen die die SSDF vorzugehen hatte, die neu formierte SPLM/A. In der Tat entwickelt sich die SSDF schnell zu einem Sammelbecken aller von Khartum gegen die SPLM/A unterstützten Milizen. Als Erste schließt sich die primär in Upper Nile verortete SSPLM-United von Lam Akol mit einem eigenen Abkommen, dem im September 1997 unterzeichneten Fashoda Agreement ${ }^{4}$, dem Prozess an. In weiterer Folge gliedern sich unterschiedliche Nuer-Milizen in Unity State in die SSPF ein. Eine wichtige Rolle spielen die von Paulino Matip und Peter Gadet kontrollierten Kräfte, die die für Khartum essenzielle Funktion der Kontrolle der Ölfelder um Bentiu übernehmen.

Die SSDF formiert sich von Beginn nicht als eine einheitliche Organisation, und sie wäre selbst als loses Sammelbecken ohne die Kooperation mit dem

3 https://www.peaceagreements.org/viewmasterdocument/550.

4 https://www.peaceagreements.org/viewmasterdocument/1039. 
Regime in Khartum nie haltbar gewesen. Als Gesamtes zeigt sie sich ineffektiv und zersplittert, was noch dadurch verstärkt wird, dass Khartum seine Unterstützungsleistungen über die einzelnen, untereinander konkurrierenden Milizen abwickelt. Auch wenn Khartum seine Gunst vom Bekenntnis zu den SSDF abhängig macht, erfährt die Organisation als Ganzes keine Unterstützung. Diesem Trend zur Ethnopolitisierung des Konfliktes kann sich wiederum die SPLM/A nicht entziehen. Sie setzt vor allem in Bahr el-Ghazal ebenfalls auf Miliz-Strukturen der Dinka-Gemeinschaften, womit sie ihr ursprüngliches Credo des geordneten Auftretens als einheitliche Organisation und der Überwindung ethnopolitischer Gräben unterläuft.

Dennoch befindet sich die SPLM/A im Aufschwung, wobei sich dieser, im markanten Unterschied zur ersten Dekade ihrer Existenz, vor allem auf Equatoria konzentriert. Dies ist nicht zuletzt durch sich wandelnde regionalund geopolitische Faktoren bedingt. So forcieren die USA ab Mitte der 1990erJahre ihre Kooperation mit der ugandischen Armee gegen die in Norduganda und den Equatorias operierende LRA, die wiederum von Khartum als Vehikel gegen die SPLM/A unterstützt wird. Diese Konstellation resultiert, historisch zum ersten Mal, in einer substanziellen militärischen Zusammenarbeit der ugandischen Armee mit der SPLM/A (LeRiche und Arnold, 2013: 102; Rolandsen, 2005: 164). Die neu gewonnene militärische Stärke entwickelt politische Strahlkraft. Nominell mit Machar verbündete Einheiten der SSIM/A stellen ihre Angriffe auf die SPLM/A ein und agieren komplementär gegen die sudanesische Armee (Johnson, 2016a: 103). Derartige informelle Kooperationen tragen wesentlich dazu bei, dass der südliche Teil von Equatoria Ende der 1990er-Jahre unter solider Kontrolle der SPLM/A steht.

Zwei weitere strategische Schritte ebnen den Weg der SPLM/A zu einer Anfang der 2000er-Jahre dominanten Position. Ab dem Jahr 1997 startet sie eine überaus erfolgreiche militärische Kampagne, »Operation Thunderbolt«. Die Kampagne beginnt zunächst in Bahr el-Ghazal und bewegt sich in der Folge weiter bis zum westlichen Nilufer. Insbesondere in Lakes und Warrap, den am Nil gelegenen Teilen von Greater Bahr el-Ghazal, zeitigt die unter dem Kommando von Salva Kiir stehende Operation bedeutende Erfolge. Strategisch wichtige Städte wie Rumbek, Warrap und Tonj werden eingenommen. Am Ende der Kampagne wird Greater Bahr el-Ghazal mit Ausnahme der Hauptstadt Wau vollständig kontrolliert. Der in Tonj geborene enge GarangVertraute Nhial Deng Nhial wird als Militärgouverneur der Region eingesetzt (Abol Kuyok, 2015: 686-687). 
Die neu errungene Stärke in Bahr el-Ghazal verdankt die SPLM/A ihrem neuen Ansatz der Einbeziehung der Gemeinschaften und der traditionellen Autoritäten. Diese Kollaboration funktioniert nicht nur in Mobilisierungen und der Organisation einer zivilen Verwaltung, sondern auch in Friedensprozessen. Eine historisch einschneidende Rolle spielt die »Wunlit Peace and Reconciliation Conference« im März 1999. Die Konferenz markiert eine Wasserscheide in der Aussöhnung von nicht zuletzt durch die entgegengesetzten politisch-militärischen Mobilisierungen verfeindeten Gemeinschaften der Dinka und Nuer (Bradbury et al., 2006: 41-52). Die Initiative zu dieser Konferenz geht nicht von der SPLM/A selbst aus. Die Vorarbeit unternimmt das New Sudan Council of Churches (NSCC), eine Dachorganisation der südsudanesischen Kirchen, die auf die Beibehaltung einer kritischen Distanz zur SPLM/A bedacht ist. Die zentrale Verbindungsperson ist der in Yirol geborene Telar Ring Deng, selbst kurz Mitglied der SPLM/A-Nasir, später wieder Teil der Torit-Fraktion, im Rahmen derer er als Generalsekretär der South Sudanese Law Society fungiert (Abol Kuyok, 2015: 716). ${ }^{5}$

Der für die Konferenz entwickelte "people-to-people«-Ansatz basiert auf dem Ausschluss der organisierten bewaffneten Kräfte. Dieser Ausschluss wird konsequent durchgesetzt, was dazu führt, dass einer von Machar entsandten SSIM/A-Delegation die Teilnahme verweigert wird. Die 360 Delegierten, neben traditionellen Autoritäten auch Repräsentant:innen von Frauen- und Jugendgruppen, konzentrieren sich in ihren Diskussionen auf konkrete Herausforderungen wie Entführungen, Grenzen zwischen den Territorien der Gemeinschaften, sowie Weiderouten und Weidegebiete. Das abschließende Wunlint Covenant ${ }^{6}$ richtet unterschiedliche Arbeitsgruppen ein, die sich diesen Streitfragen vertiefend widmen und die Implementierung von Lösungsansätzen überwachen. Der Prozess wird von Garang und Machar explizit anerkannt. Wenngleich Wunlit nicht von der SPLM/A selbst betrieben wird, erweist sich der Prozess für sie als überaus funktionell. Wunlit öffnet das Tor zur nachfolgenden SPLM/A-Dominanz in Bahr el-Ghazal und ebnet den Weg zur letztendlichen Reintegration von Riek Machar in die SPLM/A-Mehrheitsfraktion.

Zugleich verstärkt die SPLM/A ihr politisches und militärisches Engagement im Norden. Im Rahmen der NDA ist insbesondere das Bündnis mit dem ben dieser Zeilen, am 27. Dezember 2020, der Covid-19-Pandemie zum Opfer fällt. https://www.peaceagreements.org/viewmasterdocument/1813. 
im Ostsudan operierenden Beja Congress von Bedeutung, der von ihr militärisch unterstützt wird. Durch diese Unterstützung ist die SPLA in Kämpfe mit den SAF im Grenzgebiet zu Eritrea, insbesondere in der Gegend um Kassala, involviert. Dort kann sie um das Jahr 2000 einige militärische Erfolge erzielen.

Mit dem Erteilen dieser für den Norden schmerzhaften Nadelstiche geht ein interner Einigungsprozess einher. Riek Machar verlässt die SSDF, die sich in weiterer Folge unter dem Kommando von Paulino Matip auf den Schutz der Ölfelder in Unity State konzentriert (ebd.: 696). Er gründet kurzfristig die Sudan People's Defence Forces (SPDF), die ihm aber letztlich nur als Instrument zur Verhandlung seiner Reintegration in die SPLM/A dienen. Die Reintegration verläuft in der Tat zügig und wird im Januar 2002 abgeschlossen. Riek Machar ist nun wieder nominell die Nummer Drei jener Organisation, die er elf Jahre zuvor im Disput verlassen hat. Auch Lam Akol schließt sich mit seiner SPLM-Unity im Jahr 2004 wieder der SPLM/A an.

Von einer durchschlagenden militärischen Dominanz kann dennoch auf keiner der beiden Seiten gesprochen werden. Die SAF und die mit ihnen verbündeten Milizen kontrollieren nach wie vor die drei wesentlichsten Städte Juba, Wau und Malakal, in denen sich ihre militärische Schlagkraft bündelt. Die SSDF-Milizen in Unity State sind ebenfalls ein relevanter Faktor auf nordsudanesischer Seite. Im Sinne eines Vergleichs der militärischen Kräfte waren damit Anfang der 2000er-Jahre jene Bedingungen erfüllt, die I. William Zartman (2000) als ein »mutually hurting stalemate« bezeichnet hat, das im Sinne von Zartmans Begriff der »ripeness« - reif für eine Friedenslösung wäre. Dieses beide Seiten schmerzende Kräftegleichgewicht hätte theoretisch allerdings schon Mitte der 1980er-Jahre festgestellt werden können. Dies unterstreicht, dass es nicht allein für das wechselseitige Interesse an Friedensverhandlungen und deren letztendlichen Erfolg verantwortlich zu machen ist.

\section{Ein umfassendes Friedensabkommen}

Es sind zwei weitere Faktoren, einer ökonomisch, einer geopolitisch, die insbesondere die sudanesische Seite zum Eintritt in ernsthafte Verhandlungen motivieren. Der ökonomische Faktor, der schon zuvor den militärstrategischen Zugang Khartums bestimmt, ist die Ölförderung. In der zweiten Hälfte der 1990er-Jahre nahm die Ölexploration, vor allem in der Region um Bentiu, massiv zu. Im Jahr 1999 wird endlich die lange erwartete Pipeline von den Öl- 